\title{
Problemas éticos vivenciados por profissionais de saúde na assistência à gestante: revisão integrativa
}

\author{
Ethical problems experienced by health professionals in assisting pregnant women: \\ integrative review
}

\section{Problemas éticos experimentados por profesionales de salud en la asistencia a la mujer embarazada: revisión integradora}

Hianca de Souza Sodré ${ }^{*}$, Deisy Vital dos Santos ${ }^{1}$, Marla Niag dos Santos Rocha ${ }^{1}$, Sibele de Oliveira Tozetto Klein ${ }^{1}$.

\section{RESUMO}

Objetivo: Identificar os problemas éticos (PEs) vivenciados pelos profissionais de saúde na assistência obstétrica. Métodos: Trata-se de uma revisão integrativa da literatura, incluindo as bases de dados da Literatura Latino-Americana e do Caribe em Ciências da Saúde (Lilacs) e Medical Literature Analysis and Retrieval System Online (Medline), Banco de Dados em Enfermagem (BDENF), Bibliografia Nacional em Ciencias de la Salud Argentina (BINACIS) e Índice Bibliográfico Español en Ciencias de la Salud (IBECS). A amostra final foi composta por oito artigos. Resultados: Identificou-se a existência de três categorias de PEs: Nas relações entre os profissionais e as gestantes, associados à desinformação, desrespeito a autonomia e pré-julgamento; Na relação entre a equipe de saúde, davam-se no processo de trabalho e nas relações interprofissionais; e Na relação com o serviço de saúde decorreram da falta de estruturação e de serviços específicos, de apoio institucional para resolução das demandas de trabalho, além de discrepância na assistência prestada nos serviços públicos e privados. Considerações finais: Foram identificados diversos PEs envoltos na assistência obstétrica, todavia os mais representativos estiveram associados aos problemas na relação dos profissionais de saúde com as gestantes.

Palavras-chave: Bioética, Ética, Profissionais de saúde, Gravidez.

\begin{abstract}
Objective: Identify ethical problems (EPs) experienced by health professionals in obstetric care. Methods: This is an Integrative literature review, including the databases of Latin American and Caribbean Literature in Health Sciences (Lilacs) and Medical Literature Analysis and Retrieval System Online (Medline), Database in Nursing (BDENF), National Bibliography in Ciencias de la Salud Argentina (BINACIS) and Bibliographic Index Español en Ciencias de la Salud (IBECS). The final sample consisted of eight articles. Results: The existence of three categories of NPs was identified: In the relationships between professionals and pregnant women, associated with misinformation, disrespect for autonomy and pre-judgment; In the relationship between the health team, they took place in the work process and in interprofessional relationships; e In the relationship with the health service, they resulted from the lack of structure and specific services, institutional support for solving work demands, in addition to discrepancy in the care provided in public and private services. Final considerations: Several NPs involved in obstetric care were identified, but the most representative were associated with problems in the relationship between health professionals and pregnant women.
\end{abstract}

Keywords: Bioethics, Ethics, Health personnel, Pregnancy.

\section{RESUMEN}

Objetivo: Identificación de problemas éticos (PEs) vivenciados por los profesionales de salud en la asistencia obstétrica. Métodos: Se trata de una revisión integradora de la literatura, incluyendo las bases de datos de Literatura Latinoamericana y del Caribe en Ciencias de la Salud (Lilas) y Sistema de Análisis y Recuperación de Literatura Médica en Línea (Medline), Base de Datos en Enfermería (BDENF), Bibliografía Nacional en Ciencias de la Salud Argentina (BINACIS) e Índice Bibliográfico Español en Ciencias de la Salud (IBECS). La muestra final fue formada por ocho artículos. Resultados: Se identificó la existencia de tres categorías de EP: En las relaciones entre los profesionales y las mujeres embarazadas, asociados a la desinformación, falta de respecto a la autonomía y prejuicios; En la relación entre el equipo de salud, se hacían presentes en el proceso de trabajo y en las relaciones interprofesionales; y e En la relación con el servicio de salud, resultaron de la falta de estructura y servicios específicos, apoyo institucional para la solución de demandas laborales, además de la discrepancia en la atención brindada en los servicios públicos y privados. Consideraciones finales: Se identificaron varios PE involucrados en la atención obstétrica, todos los más representativos los problemas en la relación de los profesionales con las mujeres embarazadas.

Palabras clave: Bioética, Ética, Profesionales de salud, Embarazo.

\footnotetext{
${ }^{1}$ Universidade Federal do Recôncavo da Bahia (UFRB), Santo António de Jesus - BA.
}

*E-mail: hiianca.sodre@hotmail.com 


\section{INTRODUÇÃO}

A assistência obstétrica se fundamenta nos cuidados à gestante e ao feto durante o período grávidopuerperal (REZENDE J e BRAGA A, 2017). Ela deve ser realizada por uma equipe multiprofissional, envolvendo médicos, enfermeiras, parteiras, auxiliares de enfermagem e agentes de saúde (WORD HEALTH ORGANIZATION (WHO), 2016).

$\mathrm{Na}$ assistência à gestante essa equipe é responsável pelo desenvolvimento de um plano individualizado, embasado na identificação de problemas e necessidades. Nessa direção, a equipe de enfermagem, por exemplo, deve realizar o acolhimento e atualizar as informações sobre o período gestacional, enquanto os agentes de saúde devem aproximar essas mulheres aos serviços de saúde, mediando as ações (VIEIRA SM, et al., 2011).

Os profissionais envolvidos na assistência obstétrica devem oferecer um cuidado qualificado, a fim de assegurar melhores condições de saúde. Eles são importantes no processo de avaliação e comunicação sobre os processos fisiológicos, biomédicos, comportamentais e socioculturais (WHO, 2016).

Compreende-se que nesta assistência exista garantia da integralidade e da singularidade do cuidado, possibilitando uma vivência mediada pelo respeito à autonomia e proteção da mulher e da criança (MINISTÉRIO DA SAÚDE, 2017).

Para tanto, ao discutir questões referentes à relação profissional-usuário, é necessário compreender que se trata de um vínculo de confiança, dada a importância da parceria na tomada de decisão conjunta. Assim, os profissionais de saúde devem prestar informações as usuárias e estimular a compreensão dos problemas de saúde para que sejam capazes de avaliar as implicações dos tratamentos e, portanto, escolhê-los mediante suas próprias crenças e valores (HU LY, 2016).

A efetividade da relação entre usuários e prestadores de cuidados de saúde normalmente se baseia em práticas profissionais orientadas por princípios éticos. Logo, a ética é uma ciência que surge para nortear as ações humanas, fomentando a reflexão sobre valores e princípios através de diretrizes fundamentadas em padrões morais avaliados para orientar o exercício das profissões (DIAS FA, et al., 2018).

De acordo com Zugaib M, et al. (2016), a necessidade de uma assistência apropriada à gestante é crescente, como se notifica pelo conjunto de especialidades que a apoia (a exemplo da pediatria e clínica médica) e a prestação de serviços complexa, devido aos aspectos sociais, culturais, nutricionais e emocionais do período gravídico-puerperal, exigindo dos profissionais da saúde qualificação, interesse, experiência e conhecimento técnico.

Todavia, alguns estudos sinalizam que na relação estabelecida entre os profissionais e as usuárias gestantes, o desrespeito à autonomia muitas vezes é justificado com base nos princípios da beneficência e não-maleficência (DE VRIES R, 2017).

As mulheres pertencem a um grupo de grande vulnerabilidade social, decorrente de aspectos biológicos e de desigualdade de gênero (DURAN-PALACIO NM, 2015). Em consequência disso, a assistência tem mostrado falhas, podendo ser em decorrência da desinformação e insuficiente educação em saúde ou até mesmo falta de acesso aos serviços de atenção à saúde reprodutiva, resultando em problemas éticos (PEs) (NORA CRD, et al., 2015).

Assim, há uma deficiência sistêmica no cuidado à gestação, visto que muitas usuárias são desassistidas quanto às suas necessidades e ao seu bem-estar físico, psíquico e social (DE VRIES R, 2017). De todo modo, diante dos conflitos de valores e deveres e da ausência de regras prontamente estabelecidas, os PEs podem gerar diversos desfechos, sendo necessário a investigação de diferentes possibilidades. Para tanto, exigem muita cautela no processo de decisão e na pós deliberação, a fim de apontar a melhor solução para o problema (NORA CRD, et al., 2015).

Diante desta realidade, este estudo torna-se relevante, pois tem como objetivo identificar problemas éticos (PEs) vivenciados pelos profissionais de saúde na assistência à gestante, com a expectativa de apresentar evidências que estimulem mudanças e melhorias nessas relações. 


\section{MÉTODOS}

Trata-se de uma revisão integrativa de literatura (MENDES KDS, et al., 2008), realizada em duas coletas, a primeira coleta entre abril-maio de 2020 e a segunda em agosto de 2021. Foram utilizados os portais Pubmed, Scielo e da BVS, incluindo as bases de dados da Lilacs e Medline, BDENF, BINACIS e IBECS.

Os descritores de busca empregados em todas as bases foram Bioethics e Pregnancy. Assim, foram incluídos artigos publicados no idioma português, inglês e espanhol, cujos títulos e/ou resumos contemplassem aspectos relativos aos PEs vivenciados pelos profissionais de saúde na assistência à gestante, e estivessem disponibilizados na íntegra, gratuitamente. Foram excluídos os editoriais, cartas ao editor, artigos de revisão e publicações duplicadas. Com a intenção de ampliar o número de artigos, considerando a escassez de produção sobre a temática, não foi estabelecido recorte temporal. Também foi realizada a busca reversa, quando através de uma verificação manual das referências dos estudos selecionados no primeiro momento da coleta buscou-se novas referências.

Para categorização dos estudos, a partir do delineamento das informações detectadas, foi utilizado um instrumento, composto por itens relativos à identificação do artigo, periódico de publicação, objetivos, tipo de estudo e participantes da pesquisa. As categorias analíticas foram definidas a partir da adaptação da classificação utilizada em outros estudos sobre PEs, a saber: problemas éticos na relação com as gestantes, na relação entre a equipe e na relação com o serviço de saúde (NORA CRD, et al., 2015).

\section{RESULTADOS E DISCUSSÂO}

Dos 576 estudos encontrados na fase de identificação, oito (8) compuseram a amostra final desta pesquisa (Figura 1). Sete (7) das referências elegidas estavam em formato de artigos e um (1) no modelo de dissertação (Quadro 1).

Figura 1 - Fluxograma do processo de seleção dos artigos da revisão, segundo PRISMA

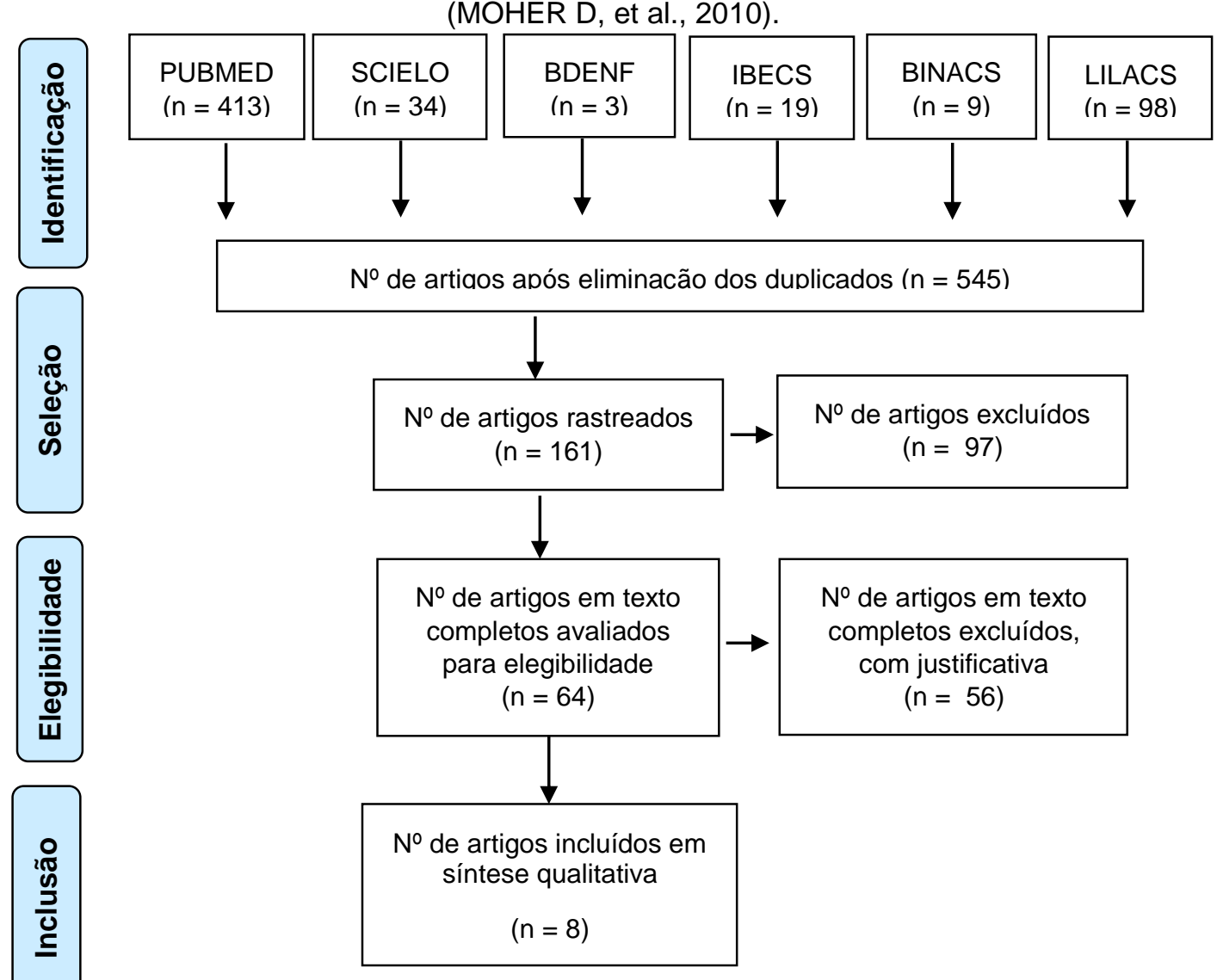

Fonte: Sodré HS, et al., 2021. 
Quadro 1 - Descrição dos estudos incluídos na revisão integrativa.

\begin{tabular}{|c|c|c|c|c|}
\hline Autores e ano & Periódico & Objetivos do estudo & Tipo de estudo & Participantes da pesquisa \\
\hline $\begin{array}{l}\text { ROCHA WB, et } \\
\text { al. (2015). }\end{array}$ & Revista Bioética & $\begin{array}{l}\text { Identificar o conhecimento e a percepção dos aspectos éticos } \\
\text { envolvidos no Programa de Interrupção Gestacional Prevista } \\
\text { em Lei (PIGL), por parte de profissionais de saúde do Hospital } \\
\text { Materno Infantil de Brasília (HMIB). }\end{array}$ & $\begin{array}{l}\text { Estudo quanti-qualitativo, do } \\
\text { tipo exploratório-descritivo. }\end{array}$ & $\begin{array}{l}177 \text { profissionais de saúde (médicos (35), assistentes } \\
\text { sociais (5), psicólogos (9), farmacêuticos/bioquímicos } \\
\text { (8), enfermeiros (68) e técnicos de enfermagem (52), } \\
\text { todos eles atuando no HMIB e sem vínculos diretos com } \\
\text { PIGL). }\end{array}$ \\
\hline $\begin{array}{l}\text { URREA F } \\
\text { (2014). }\end{array}$ & $\begin{array}{l}\text { Colombiana de } \\
\text { Bioética }\end{array}$ & $\begin{array}{l}\text { Compreender os significados } \\
\text { que as mulheres grávidas e os profissionais de saúde têm em } \\
\text { relação ao corpo da mulher grávida e sua relação com o } \\
\text { exercício da autonomia no espaço da assistência no hospital de } \\
\text { Suba, Usaquén y Engativá, do Norte da Secretaria Distrital de } \\
\text { Saúde de Bogotá. }\end{array}$ & $\begin{array}{l}\text { Qualitativo, com entrevista } \\
\text { semiestruturada }\end{array}$ & $\begin{array}{l}10 \text { profissionais de saúde (odontólogos (3), médicos (3) } \\
\text { e enfermeiros (4)). }\end{array}$ \\
\hline $\begin{array}{l}\text { ATZINGEN ACV } \\
\text { (2012). }\end{array}$ & Revista Bioética & $\begin{array}{l}\text { Conhecer a relação radiologista-usuário durante os exames de } \\
\text { ultrassonografia obstétrica sob os pontos de vista materno e do } \\
\text { radiologista. }\end{array}$ & $\begin{array}{l}\text { Qualitativo, exploratória e } \\
\text { transversal. }\end{array}$ & $\begin{array}{l}10 \text { radiologistas que realizavam ultrassonografia } \\
\text { obstétrica. }\end{array}$ \\
\hline $\begin{array}{l}\text { ISRAEL GR } \\
(2002) \text {. }\end{array}$ & $\begin{array}{l}\text { Dissertação de } \\
\text { Mestrado }\end{array}$ & $\begin{array}{l}\text { Compreender as implicações éticas da oferta do teste rápido } \\
\text { anti-HIV a gestantes em trabalho de parto }\end{array}$ & Qualitativo. & $\begin{array}{l}8 \text { profissionais de saúde (enfermeiros (7) e auxiliar de } \\
\text { enfermagem (1)). }\end{array}$ \\
\hline $\begin{array}{l}\text { HEGER MA, et } \\
\text { al. (2018). }\end{array}$ & Revista Acta Bioethic & $\begin{array}{l}\text { Investigar a experiência de mulheres que conceberam crianças } \\
\text { intra-uterinas incompatíveis com a vida ao nascer, através da } \\
\text { história de três mulheres que passaram por essa situação e oito } \\
\text { informantes qualificados. }\end{array}$ & $\begin{array}{l}\text { Abordagem qualitativa e de } \\
\text { alcance exploratório. }\end{array}$ & 8 profissionais de saúde (parteiras (7) e psicóloga (1)). \\
\hline $\begin{array}{l}\text { NGAN OMY, et } \\
\text { al. (2018). }\end{array}$ & $\begin{array}{l}\text { BMC Health Services } \\
\text { Research }\end{array}$ & $\begin{array}{l}\text { Identificar os fatores que influenciam a atuação do prestador, } \\
\text { como consulta e encaminhamento, na prestação de serviços do } \\
\text { teste de pré-natal não invasivo (NIPT) para Síndrome de Down } \\
\text { nos setores público e privado de saúde. }\end{array}$ & $\begin{array}{l}\text { Qualitativo transversal, com } \\
\text { entrevista semiestruturada }\end{array}$ & 20 profissionais de saúde (obstetras (8) e parteiras (12)). \\
\hline $\begin{array}{l}\text { NGAN OMY, et } \\
\text { al. (2017). }\end{array}$ & $\begin{array}{l}\text { BMC Pregnancy and } \\
\text { Childbirth }\end{array}$ & $\begin{array}{l}\text { Explorar a visão de profissionais obstétricos em Hong Kong } \\
\text { sobre a utilidade clínica do NIPT em comparação com a triagem } \\
\text { atual da Síndrome de Down e as práticas de diagnóstico e } \\
\text { preocupações éticas que possam surgir com o uso do NIPT. }\end{array}$ & $\begin{array}{l}\text { Qualitativo transversal, por } \\
\text { meio de questionário. }\end{array}$ & $\begin{array}{l}327 \text { profissionais obstétricos (parteiras (237) e obstetras } \\
(90)) \text {. }\end{array}$ \\
\hline $\begin{array}{l}\text { MENDES RWM, } \\
\text { et al. (2020). }\end{array}$ & Revista Femina & $\begin{array}{l}\text { Verificar a existência e os motivos da objeção de consciência } \\
\text { na atençãa integral à saúde das vítimas de violência sexual, } \\
\text { com gravidez indesejada e com desejo de interrupção da } \\
\text { gestação, no estado de Minas Gerais. Também se objetiva } \\
\text { conhecer a estrutura de atendimento das instituições } \\
\text { credenciadas na rede de atenção à vítima de violência sexual, } \\
\text { verificar a frequência de objeção de consciência nesses } \\
\text { serviços e identificar como a unidade procede em relação a } \\
\text { esse problema na resolução do aborto legal e na atenção } \\
\text { integral à mulher vítima de violelencia sexual. }\end{array}$ & $\begin{array}{l}\text { Quantitativo, transversal, } \\
\text { descritivo e analítico. }\end{array}$ & $\begin{array}{l}35 \text { instituições credenciadas pela Secretaria de Estado } \\
\text { de Saúde de Minas Gerais (SES/MG). }\end{array}$ \\
\hline
\end{tabular}

Fonte: Sodré HS, et al., 2021.

REAS | Vol.13(9) | DOI: https://doi.org/10.25248/REAS.e8638.2021 Página 4 de 9


Assim, a partir das categorias analíticas definidas, os resultados e discussões foram apresentados nos seguintes agrupamentos: problemas éticos nas relações com as gestantes, problemas éticos nas relações entre a equipe e problemas éticos nas relações com o sistema de saúde.

Em relação ao país de publicação, quatro artigos são brasileiros, dois são chineses, um colombiano e um chileno. Quanto ao ano de publicação, as produções aconteceram entre os anos de 2002 à 2020, sendo duas publicações do ano de 2018. Todos os estudos utilizaram como metodologia a abordagem qualitativa, dentre os participantes estiveram profissionais médicos, enfermeiras, técnicos de enfermagem, assistentes sociais, psicólogos, farmacêuticos/bioquímicos, odontólogos e parteiras.

\section{Problemas éticos nas relações com as gestantes}

Nessa categoria são apresentados os PEs vivenciados pelos profissionais de saúde durante a assistência à gestante, incluindo problemas referentes à comunicação, desrespeito à autonomia da gestante e préjulgamento. Nos problemas observados, houve elevada frequência de problemas associados à desinformação.

A expressiva presença de PEs vivenciados por profissionais de saúde na relação com a gestante pode estar relacionada com a imprevisibilidade das ocorrências resultantes das relações humanas, existindo uma interseção entre a demanda dos usuários e a prestação dos serviços por profissionais ainda inabilitados. Assim, um dos estudos dessa revisão mostrou que muitas vezes as práticas são motivadas por técnicas, procedimentos e rotinas "paternalistas" que ditam o que creem ser "melhor" para a usuária (HEGER MA, et al., 2018).

Nessa direção, verifica-se o "paternalismo médico", condição em que o médico está convicto de que a administração e tomada de decisão da usuária deve ser realizada somente por profissional qualificado, não possibilitando que a mesma realize a auto gerência de seus cuidados (GRACIA D, 2002).

Gracia D (2002) afirma que as grandes relações sociais da cultura ocidental se desenvolveram baseadas no paternalismo até o final do século $X X$, inclusive a política. Logo, esse foi um dos cenários fundamentais para o desenvolvimento da bioética, visto que a gestão dos corpos, abrangendo a sexualidade, vida e morte estavam sob a responsabilidade e controle de médicos, governantes e técnicos treinados.

Em consonância, um dos artigos selecionados identificou a ausência de esclarecimento quanto às indicações, resultados e o destino do exame ultrassonográfico obstétrico por parte dos radiologistas (ATZINGEN ACV, 2012). Além disso, outro estudo dessa revisão verificou que muitos profissionais optaram por omitir ou abreviar as informações como forma de serem mais bem compreendidos ou a fim de obter maior adesão ao procedimento (NGAN OMY, et al., 2018). Entretanto, essa prática pode fomentar a falsa impressão de proximidade na relação profissional-usuário, sendo crucial que o profissional preste informações claras, adequadas e ausentes de coerção (GRACIA D, 2002).

Outro artigo dessa revisão identificou que os direitos de autonomia das gestantes são incompatíveis com os discursos dos profissionais de saúde, que argumentam que estas sempre estão sob influência do marido ou da mãe (ATZINGEN ACV, 2012). Mas é importante considerar que a inserção da parceria sexual no processo, não afasta a autonomia da mulher, pelo contrário, se mostra como uma possibilidade de apoio e companhia, caso seja o desejo da gestante (TEJEDOR JM, et al., 2015).

O Manual de Ética em Ginecologia e Obstetrícia garante os direitos das gestantes no atendimento obstétrico, reconhecendo, dentre eles, o direito à autonomia em discordar das indicações médicas e ir a busca de uma segunda opinião ou parecer de outro médico, à informação e ao respeito (BOYACIYAN K, 2011).

O pré-julgamento das gestantes por parte da equipe, com relação a desconfiança quanto à veracidade das informações prestadas e a utilização do conhecimento biomédico dos profissionais também foi verificado como um PE e um método de dominação para subjulgar as gestantes (ATZINGEN ACV, 2012). Giugliani C, et al. (2019) ressalta que é de fundamental importância que os profissionais de saúde proporcionem um atendimento acolhedor e sem julgamentos, pois estes podem fazer com que muitas mulheres deixem de compartilhar sua situação. 
Um estudo dessa revisão mostrou que $60,6 \%$ das instituições pesquisadas violaram o direito da gestante ao abortamento legal, mediadas pela objeção de consciência de toda equipe (MENDES RWM, et al., 2020). Nessa direção, a bioética tem como objeto de estudo a vida e seus fatores éticos, e ao longo dos anos tem passado por diferentes fases, com enfretamento de diversas questões, como autogestão do corpo, autonomia, distribuição de recursos e princípio da justiça. Além dos problemas referentes à globalização e direitos das gerações futuras (GRACIA D, 2002).

Para tanto, como afirma Diniz D e Guilhem D (2009), há uma necessidade da bioética se mostrar comprometida com a opressão social e as desigualdades, pois a neutralidade das perspectivas bioéticas pode enveredar-se para a validação da hegemonia de cada sociedade.

\section{Problemas éticos nas relações entre as equipes de trabalho}

A fragilidade e os $\mathrm{PEs}$ existentes entre os profissionais de saúde que compõem as equipes de assistência às gestantes podem estar associados ao processo de trabalho e as relações interprofissionais. Quanto ao processo de trabalho, os problemas mais relevantes foram decorrentes da dificuldade na delimitação de papéis e responsabilidades de cada profissional. As diferenças nas formações acadêmicas, experiências e o surgimento de novas técnicas no trabalho assistencial podem levar ao surgimento desses PEs, exigindo dos profissionais o desprendimento e esforço para respeitar essas diferenças (NORA CRD, et al., 2015).

Um artigo dessa revisão demonstrou que profissionais que prestam assistência secundária, como radiologistas, sentem-se desorientados quanto à revelação de diagnósticos, já que acreditam ser dever do profissional solicitante, se configurando, portanto, como causa de discórdia entre a equipe (ISRAEL GR, 2002). Sobre a singularidade do trabalho dos radiologistas, Otoni JC, et al. (2018) afirmam que, predominantemente, a responsabilidade destes profissionais tem se restringido ao relatório escrito como ferramenta de comunicação sobre resultados de exames, acreditando ser dever do médico solicitante a comunicação e apresentação dos resultados a usuária, já que é a pessoa a quem essa tem mais acesso (COX J e GRAHAM Y, 2019).

Entretanto, os departamentos de radiologia têm reconhecido a necessidade do estabelecimento de uma comunicação mais frequente e facilitada, por isto realizam estudos para a reformulação de processos e protocolos correntes, bem como, a definição de práticas e mudança cultural relativos a estes procedimentos (SMITH JDR, 2014).

A discriminação aos profissionais que atuam no programa de abortamento legal também foi verificada em um estudo dessa revisão, demonstrando conflitos destes profissionais com outros de demais setores do serviço de saúde (URREA F, 2014; MENDES RWM, et al., 2020).

Nessa direção, os profissionais de saúde do programa de abortamento legal possuem além da motivação moral e religiosa, a discriminação que existe entre os profissionais, fazendo com que temam serem reconhecidos como "aborteiros" pela equipe (MADEIRO AP e DINIZ D, 2016).

Godoi AMM e Garrafa V (2014) afirmam que os aspectos sociais envolvidos no processo de estigmatização presente nas relações interpessoais têm fortes implicações nas discussões bioéticas, pois exige a investigação das estruturas sociais, sob uma perspectiva macro, a fim de identificar e avaliar as suas consequências para a saúde. Isso porque o estigma pode ampliar a vulnerabilidade de indivíduos e grupos e repercutir sobre as condições de saúde do profissional, que é reprimido pelas expressões negativas.

Em outra investigação, o PE esteve associado ao questionamento por parte de alguns profissionais acerca de uma solicitação de exame, ou seja, alguns membros da equipe de enfermagem atuantes numa maternidade refutaram a solicitação de realização de teste anti-HIV numa gestante tatuada que já havia realizado seu exame durante o pré-natal (HEGER MA, et al., 2018). Compreende-se que a discussão de atribuições e responsabilidades é uma prática que deve estar intensamente presente na equipe multiprofissional, a fim de não haver comprometimento à assistência integral e respeito às competências de cada profissão, evitando possíveis conflitos dentro da equipe de trabalho (SIQUEIRA-BATISTA R, et al., 2015). 
Logo, valer-se de métodos deliberativos que possibilitam a análise e mudança de perspectivas de maneira contínua, moderada e não coercivo é um procedimento fundamental nas relações interpessoais, principalmente, quando se trata de valores relacionados à saúde das pessoas. Desse modo, a bioética deve ser deliberativa e deve ainda desempenhar seu papel em favor da vida humana, tanto para o indivíduo quanto para uma equipe de trabalho (GRACIA D, 2009).

\section{Problemas éticos nas relações com o sistema de saúde}

Os PEs que mais se destacaram nessa categoria são decorrentes do desconhecimento ou ausência de protocolos que norteiem a prática dos profissionais na assistência e serviços, por exemplo, a falta de estruturação e de serviços específicos, bem como de apoio institucional para resolução das demandas de trabalho, além de discrepância na assistência prestada nos serviços públicos e privados (MENDES RWM, et al., 2020; NGAN OMY, et al., 2018; NGAN OMY, et al., 2017).

Os protocolos podem se mostrar como importantes ferramentas na resolução de problemas que permeiam a assistência e a gestão dos serviços, os quais são capazes de organizar, padronizar e otimizar os serviços. Além disso, a adoção de protocolos pode fomentar o avanço e a melhoria na prestação da assistência a partir dos recursos disponíveis, principalmente, segurança do paciente (KRAUZER IM, et al., 2018).

Estudo sobre abortamento legal demostrou que quando fundamentados pela ética profissional e informados sobre a legislação, os profissionais se sentem seguros para realizar o procedimento ou fazerem uso justificado da objeção de consciência (SILVA HPG, 2014). Entretanto, as lacunas presentes na assistência à essas mulheres, como falta de estrutura física apropriada com ambiente reservado, em consonância com o direito à objeção de consciência do profissional de saúde acabam na violação do direito de abortamento legal (MENDES RWM, et al., 2020).

Um dos estudos selecionados foi realizado no Chile, onde evidenciou-se ausência de apoio institucional, falta de legitimidade e de respaldo institucional para nortear a assistência obstétrica para mães de bebês com malformações congênitas (NGAN OMY, et al., 2018). Para tanto, no Chile, a realidade vivenciada pelos profissionais é da inexistência de protocolos de ação que orientem os profissionais no manejo de gestantes com bebês diagnosticados com malformações, ficando a critério da equipe a condução dos casos. Além disso, o suporte psicológico é restrito a algumas instituições e o abortamento terapêutico não é permitido no país, 0 que pode ser resultado da precariedade de ações efetivas na assistência às gestantes de bebês com malformações (CASAS L e VIVALDI L, 2016).

No Brasil, normalmente, o cotidiano dos profissionais que cuidam de gestantes é permeado por dificuldades que envolvem os exames laboratoriais e os outros serviços fundamentais para que seja ofertado um cuidado integral ao binômio mãe-filho. Nesse contexto, apesar da expansão do acesso à assistência prénatal, ainda persistem desafios associados à efetiva realização de técnicas, como diagnóstico e acompanhamento pré-natal precoce, assistência à saúde em rede, tratamento de afecções, entre outros procedimentos que podem minimizar os desfechos desfavoráveis (VIELLAS EF, et al., 2014).

Um estudo brasileiro que investigou profissionais que acompanham gestantes soropositivas concluiu que os desafios decorrentes da demora do resultado de exames geram ansiedade, angústia ou aflição, e que estas emoções podem ter implicação direta na qualidade de vida dos membros da equipe e da assistência à gestante (SILVA O, et al., 2012).

Também foram encontradas questões referentes à submissão das mulheres grávidas a vários testes de triagem e métodos diagnósticos. Não havendo preocupação acerca das possíveis implicações sociais, como discriminação dos bebês com malformações e aborto voluntário (COX J e GRAHAM Y, 2019).

A contraposição entre sentimentos e possibilidades dada pelos diagnósticos pré-natal desafia o serviço de saúde, pois esses instrumentos se destinam à prestação de informações que situem os pais acerca da existência de malformação ou de problemas genéticos e, assim, permite que seja desenvolvido um prognóstico favorável ao feto. Todavia, pode levar a diversas questões éticas, já que a informação pode ser mal interpretada pelos pais e resultar em falsas expectativas (YOUNG MV e NUNEZ JH, 2014). 
Um estudo realizado por Silva O, et al. (2012), verificou que a falta de apoio pode fazer com que os profissionais se sintam despreparados e desguardados institucionalmente para lidar com os resultados desfavoráveis obtidos por meio dos exames diagnósticos durante o pré-natal ou até mesmo no trabalho de parto. Nesta mesma perspectiva, as gestantes podem ter sensação de desamparo, sendo forçadas a vivenciar um processo emocionalmente difícil, perpassando pelo sentimento de rejeição e sem proposta de solução pelos serviços de saúde.

A utilização das tecnologias diferenciadas nos serviços públicos e privados também podem levar à discrepâncias evidentes na assistência prestada às gestantes, pois muitas vezes, o serviço público é classificado como de baixa qualidade e destinados às pessoas pobres, enquanto que o serviço privado, é conhecido como destinado a pacientes com maior poder aquisitivo e mais eficiente por possuir exames mais elaborados e modernos (NGAN OMY, et al., 2018; NGAN OMY, et al., 2017).

Essa discrepância entre os serviços públicos e privados foi observado nos estudos realizados na China (NGAN OMY, et al., 2018; NGAN OMY, et al., 2017). O país tem apresentado importantes avanços em relação aos serviços de saúde, com expansão do seguro de saúde, mudanças nos hospitais públicos e fortalecimento da atenção primária. No entanto, a China ainda tem limitações referentes à ausência de treinamento técnico dos profissionais e de recursos financeiros destinados ao atendimento integral e de qualidade (LI L e FU H, 2017).

Para tanto, muitas correntes bioéticas podem auxiliar no manejo dos problemas presentes na relação com o serviço de saúde. A exemplo, a Bioética de Proteção que estabelece o princípio de proteção, o qual visa aplicar os deveres de responsabilidade social do Estado e atender as necessidades dos usuários, principalmente, daqueles que se encontram incapazes de garantir seu direito aos cuidados em saúde (ARAUJO FRF, 2012).

Ademais, a Bioética "Principialista" colabora, pois se baseia nos direitos universais, como respeito à autonomia, justiça, não-maleficência e beneficência, possibilitando a reflexão e julgamentos das ações (ROCHA ILS, et al., 2019). E, por se tratar de problemas originados a partir da vulnerabilidade vivenciada pela mulher, a tomada de decisão pode ser fundamentada ainda na Bioética do Cuidado, que está centrada na diferença de gênero e busca, a partir das relações humanas, a solidariedade, empatia e o respeito à dignidade humana (DURAN-PALACIO NM, 2015).

\section{CONSIDERAÇÕES FINAIS}

Essa revisão integrativa permitiu identificar três categorias de problemas éticos vivenciados por profissionais de saúde na assistência obstétrica. Todavia, a mais representativa foi dos PEs nas relações com a gestante, estando vinculados à desinformação, ao desrespeito a autonomia e o pré-julgamento das gestantes. Sugere-se: sensibilização ética dos profissionais de saúde e reestruturação dos serviços, através da criação de espaços que possibilitem uma relação dialógica entre as protagonistas desses PEs. Reconhecese como limitação dessa revisão o número reduzido de publicações sobre a temática, mesmo utilizando inúmeras estratégias para captação de novos artigos. Nessa direção, novas investigações são necessárias a fim de mapear os problemas vivenciados na perspectiva dos profissionais e das gestantes.

\section{REFERÊNCIAS}

1. ARAUJO FRF. A bioética da proteção e as implicações morais no acesso desigual ao parto: o caso de uma maternidade de referência no agreste de Pernambuco. Tese de Doutorado. Centro de Pesquisas Aggeu Magalhães. 2012.

2. ATZINGEN ACV. Relação radiologista-paciente no exame ultra-sonográfico obstétrico: o discurso do sujeito coletivo sob o enfoque bioético de gestantes e radiologistas. Revista Brasileira de Ginecologia e Obstetrícia [Internet], 2009; 31(2): 102-102.

3. BOYACIYAN K, coordenador. Ética em ginecologia e obstetrícia. 4a ed. São Paulo: CREMESP; 2011.

4. CASAS L, VIVALDI L. Embarazos incompatibles con la vida extrauterina en Chile. RedSeca: Revista de críticas políticas, sociais e culturais. 2016.

5. COX J, GRAHAM Y. Radiology and patient communication: if not now, then when?. Revista European Radiology, 2019; 30(1): 501-503. 
6. DE VRIES R. Obstetric ethics and the invisible mother. Narrative inquiry in bioethics, $2017 ; 7(3): 215-220$.

7. DIAS FA, et al. Ética, moral e processos de subjetivação: um estudo sobre a formação em psicologia, 2018.

8. DINIZ D, GUILHEM D. Bioética feminista: o resgate político do conceito de vulnerabilidade. Revista Bioética, 2009; $7(2)$.

9. DURÁN-PALACIO NM. La ética del cuidado: uma voz diferente. Rev. Fundación Universitaria Luis Amigó; 2015; 2(1): 12-21.

10. GIUGLIANI C, et al. O direito ao aborto no Brasil e a implicação da Atenção Primária à Saúde. Revista brasileira de medicina de família e comunidade, 2019; vol. 14, n. 41, 1791.

11. GODOI AMM, GARRAFA V. Leitura bioética do princípio de não discriminação e não estigmatização. Saúde Soc. [Internet], 2014; 23(1).

12. GRACIA D. De la bioética clínica a la bioética global: treinta años de evolución. Acta bioethica, 2002; 8(1): 27-39.

13. HEGER MA, et al. Vivencia de madres gestantes de niños y niñas con malformaciones incompatibles con la vida. Acta bioeth. [Internet]. 2018; 24 (1): 67-74.

14. HU LY. Professional-Patient Relationship. In: ten Have H. (eds) Encyclopedia of Global Bioethics. Springer, Cham. 2016.

15. ISRAEL GR. Como é que eu vou dizer para a gestante? Dilemas morais da oferta do teste anti-HIV a gestantes em trabalho de parto: uma contribuição da bioética. 2002. 88 f. Dissertação (Mestrado em Saúde Pública) - Escola Nacional de Saúde Pública Sergio Arouca, Fundação Oswaldo Cruz, Rio de Janeiro, 2002.

16. KRAUZER IM, et al. A construção de protocolos assistenciais no trabalho em enfermagem. Revista Mineira de Enfermagem, 2018; 22: 1-9.

17. LI L, FU H. China's health care system reform: progress and prospects. The International journal of health planning and management, 2017; 32(3), 240-253.

18. MADEIRO AP, DINIZ D. Serviços de aborto legal no Brasil-um estudo nacional. Ciência \& Saúde Coletiva, 2016; 21: 563-572.

19. MENDES KDS, et al. Revisão integrativa: método de pesquisa para a incorporação de evidências na saúde e na enfermagem. Texto \& contexto-enfermagem, 2008; 17: 758-764.

20. MENDES RWM, et al. Objeção de consciência na dinâmica do atendimento ao aborto legal em Minas Gerais. Femina, 2020; 646-653.

21. MINISTÉRIO DA SAÚDE. Manual de acolhimento e classificação de risco em obstetrícia. Secretaria de Atenção à Saúde - Brasília: Ministério da Saúde, Brasil, 2017. Disponível em: https://bvsms.saude.gov.br/bvs/publicacoes/manual_acolhimento_classificacao_risco_obstetricia_2017.pdf

22. MOHER D, et al. Preferred reporting items for systematic reviews and meta-analyses: the PRISMA statement. Int J Surg, 2010; 8(5): 336-41.

23. NGAN OMY, et al. Service provision of non-invasive prenatal testing for Down syndrome in public and private healthcare sectors: a qualitative study with obstetric providers. BMC health services research, 2018; 18(1): 731.

24. NGAN OMY, Yi H, et al. Obstetric professionals' perceptions of non-invasive prenatal testing for Down syndrome: clinical usefulness compared with existing tests and ethical implications. BMC pregnancy and childbirth, 2017; 17(1): 285.

25. NORA CRD, et al. Problemas éticos vivenciados por enfermeiros na atenção primária à saúde: revisão integrativa da literatura. Revista Gaúcha de Enfermagem, 2015; 36(1): 112-121.

26. OTONI JC, et al. Comunicação médico-paciente em exames diagnósticos: qual o papel do radiologista? Radiol Bras. 2018; 51(2): 109-111.

27. REZENDE J, BRAGA A. Conceito, Etimologia, Histórico e Obstetrícia no Brasil. In: MONTENEGRO CAB, REZENDE FILHO J. Rezende obstetrícia. 13. ed. - Rio de Janeiro : Guanabara Koogan, 2017.

28. ROCHA ILS, et al. Correntes da Bioética: Reflexões sobre a Bioética da Proteção em Saúde da Família. Brazilian Journal of Surgery and Clinical Research - BJSCR. 2019; 28(2): 39-43.

29. ROCHA WB, et al. Percepção de profissionais da saúde sobre abortamento legal. Rev. Bioét. [online]. 2015; 23(2): 387-399.

30. SILVA HPG. Aborto e objeção de consciência: aspectos éticos e legais. 2014.

31. SILVA O, et al. Trinta minutos que mudam a vida: Teste Rápido Anti-HIV Diagnóstico para parturientes e acesso ao pré-natal. Enfermagem em Foco, 2012; 3(4): 211-21.

32. SIQUEIRA-BATISTA R, et al. (Bio)ética e estratégia saúde da família: mapeando problemas. Saúde Soc. 2015; 24(1): 113-28.

33. SMITH JDR. Patient Communications: Radiology's New Imperative. Radiology Business. 2014.

34. TEJEDOR JM, et al. Aspectos éticos en la asistencia al embarazo y el parto. Ágora de enfermería, 2015; $19(2): 80-82$.

35. URREA F. Respeto a la autonomía de mujeres gestantes en salud: diálogo bioéticagénero. Revista Colombiana de Bioética, 2014; 9: 193-236.

36. VIEIRA SM, et al. Percepção das puérperas sobre a assistência prestada pela equipe de saúde no pré-natal. Texto \& Contexto - Enfermagem, 2011; 20: 255-262.

37. VIELLAS EF, et al. Assistência pré-natal no Brasil. Cadernos de Saúde Pública, 2014; 30: S85-S100.

38. WORLD HEALTH ORGANIZATION. WHO recommendations on antenatal care for a positive pregnancy experience: executive summary (No. WHO/RHR/16.12). World Health Organization. 2016.

39. YONG MV, NUNEZ JH. La bioética en el desarrollo de la ginecobstetricia. Revista Cubana de Investigaciones Biomédicas, 2014; 33(4): 431-440, 2014. In: VALERO DE BERNABÉ M, DE EUGENIO J. Medicina perinatal y diagnóstico prenatal. Cuad Bioét, 2009; 20(3): 441-50.

40. ZUGAIB M, et al. Obstetrícia: conceito e desafios. In: Zugaib M. Zugaib obstetrícia. 3. ed. Barueri, SP: Manole, 2016; p.2-7. 